\title{
Double Vision as a Presenting Symptom in an Ophthalmic Casualty Department
}

\author{
R. J. MORRIS \\ London
}

\begin{abstract}
Summary
All patients presenting with double vision as a principal symptom who presented to the Casualty Department of Moorfields Eye Hospital over a nine month period were prospectively investigated. Two hundred and seventy five patients were identified accounting for $1.4 \%$ of all casualties. Monocular diplopia was commoner than expected accounting for 69 cases $(25.1 \%)$ and was almost always a genuine symptom attributable to uniocular pathology, most commonly lenticular or corneal pathology. Two hundred and six patients $(\mathbf{7 4 . 9} \%)$ had binocular diplopia and in most cases an aetiology was established. Cranial nerve palsies were the commonest cause of biocular diplopia accounting for 81 cases. Other causes were classified as convergence or accommodation problems, decompensating phorias, traumatic, muscular, orbital lesions, supranuclear lesions, and other miscellaneous conditions. In eight cases of monocular and 23 cases of binocular diplopia no cause for the symptoms could be identified.
\end{abstract}

Diplopia is a common and distressing symptom which may be the presenting feature of a wide variety of ocular and neurological conditions. Many patients with double vision present directly to an ophthalmic accident and emergency department and the ophthalmologist is the first clinician with the opportunity to establish the diagnosis and prevent unnecessary, time consuming and expensive investigations.

Previous studies have considered the problem of diplopia, but only in selected patient populations. These have included a general ophthalmic outpatient clinic, ${ }^{1}$ orthoptic departments $^{2-4}$ and a paralytic squint clinic. ${ }^{5}$ However, these studies represent specific groups of patients and would exclude some of the common causes of diplopia. One study has considered patients presenting with diplopia to an ophthalmic emergency department, ${ }^{6}$ but did not include patients with monocular diplopia. It has been stated in the literature that monocular diplopia is rare and that a cause cannot usually be detected. ${ }^{7}$ Some authors feel that psychogenic factors are the most common cause of this symptom. ${ }^{8}$ However, there is no epidemiological information on prevalence of monocular diplopia in the literature to support these statements.

The Casualty Department of Moorfields Eye Hospital sees patients referred by their general practitioners and opticians as well as walk in casualties (self-referrals), and therefore represents a relatively unselected patient population. In order to investigate the incidence of diplopia as a presenting symptom, the proportion of patients with monocular and binocular diplopia and the aetiology of the symptom, a prospective study of consecutive patients with double vision as a principal

From: Moorfields Eye Hospital, City Road, London EC1V 2PD

Correspondence to: R. J. Morris, FRCS, FCOphth, Southampton Eye Hospital, Wilton Avenue, Southampton SO9 4XW. 
complaint who presented to the Casualty Department over a nine month period was conducted.

\section{Methods and Materials}

All patients who presented with double vision as a principal complaint to the Casualty Department, Moorfields Eye Hospital, City Road between April and November 1987 were included in the study. Patients referred by other ophthalmologists for a second opinion were excluded from the study. The majority of patients were examined by the author at presentation, but when this was not possible, the notes were reviewed and discussed with the examining ophthalmologist. All patients with binocular diplopia were also assessed by an orthoptist. When necessary patients were referred to specialist ophthalmic and neuro-ophthalmic clinics for further investigation. In some cases the patients were admitted for neurological investigation. All patients without an aetiological diagnosis were followed until their symptoms resolved.

\section{Results}

Nineteen thousand six hundred and sixty four patients attended the casualty department during the nine month period of the study. Two hundred and seventy five patients presented with diplopia as a principal symptom accounting for $1.4 \%$ of all patients. Two hundred and six patients $(74.0 \%)$ had binocular diplopia and 69 (25.1\%) monocular diplopia.

\section{Monocular Diplopia}

The causes of monocular diplopia are summarised in Table I. Extra-ocular optical causes accounted for seven patients' symptoms. Spectacle induced diplopia resulted from the bifocal segment ledge in four patients and refractive correction of myopia in one. Contact lens induced monocular diplopia occurred in two patients due to lens decentration and lens spoilage.

Ocular abnormalities were the commonest group of conditions causing monocular diplopia. Lens abnormalities accounted for 27 patients $(39.1 \%)$ and included cortical, nuclear and posterior subcapsular cataracts. Four patients in this group had decentred posterior chamber intraocular lens implants, in two the symptoms were related to optical aberrations from the edge of the lens and in two from the dialling hole. Seventeen patients $(24.6 \%)$ had corneal pathology resulting in corneal scarring, epithelial irregularity or irregular astigmatism. In addition three patients had irregular corneal astigmatism induced by central lid chalazion.

Four patients had monocular diplopia as a result of macular pathology. Two patients developed transient diplopia following trauma but ocular examination was within normal limits.

In eight $(11.6 \%)$ patients no cause for the complaint of monocular diplopia could be established. Four of these patients were lost to follow up and in four the symptoms spontaneously resolved within six weeks.

\section{Binocular Diplopia}

The causes of binocular diplopia are summarised in Table II. Infranuclear cranial nerve palsies were the commonest cause and accounted for 81 cases $(29.5 \%$ of all cases and $39.3 \%$ of binocular cases). No patient had more than one oculomotor nerve involved. Cranial nerve palsies were most commonly due to diabetes or microvascular disease ( 32 patients), although superior oblique palsies were most frequently due to longstanding congenital palsies (14 patients). Two patients with 3rd nerve palsies had orbital pseudotumours, one also had involvement of the ophthalmic nerve, and the other had an isolated palsy of the superior division of the 3rd nerve. One patient developed an isolated inferior rectus palsy following ophthalmic herpes zoster. Three patients had intracerebral tumours, one a pituitary tumour invading the cavernous sinus and two metastatic tumours. Eight patients in this group were lost to follow up.

The next most common group of patients with binocular diplopia was that classified as muscular. This included nine patients with squint, five with diplopia following cataract surgery, two adults with Duane's syndrome, two with long standing tropias who had disruption of suppression.

Twenty seven patients had a history of trauma. Fifteen had a blow out fracture and five sustained blunt orbital trauma but a frac- 
Table I. Causes of Monocular Diplopia

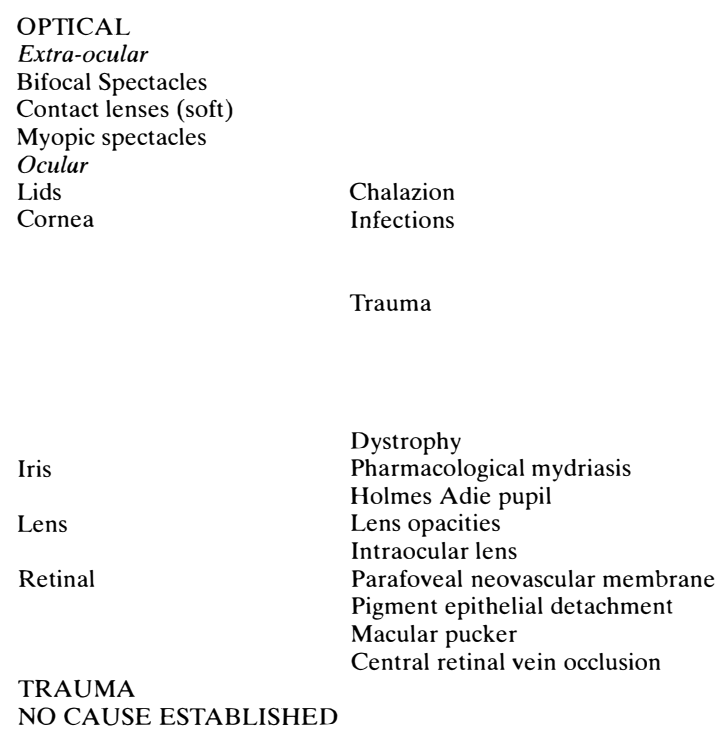

ture could not be demonstrated radiologically despite the presence of diplopia in vertical gaze positions. Two presented some days after penetrating orbital trauma and one patient developed post concussion syndrome following a closed head injury. Four patients presented following non-strabismus surgery, three had symptoms -following successful retinal detachment surgery and one following repeated pterygium surgery which had resulted in a mechanical limitation of abduction of the involved eye.

Fifteen patients had diplopia associated with supranuclear lesions. Six patients with internuclear ophthalmoplegia were subsequently diagnosed as having multiple sclerosis, four patients had transient diplopia associated with brain stem ischaemia, three had skew deviations related to cerebral ischaemia, one had migraine and one Wernicke's encephalopathy. One patient with a large pituitary tumour and bitemporal field loss presented with hemifield slip.

In 23 patients $(11.1 \%)$ no specific cause for the diplopia could be established. In 11 of these cases the symptoms had resolved within six weeks and the remaining 12 patients were lost to follow up.

\section{Discussion}

This series of patients with diplopia repre- sents the first study to investigate patients presenting to an ophthalmic emergency department with monocular and binocular diplopia. It has been suggested that monocular diplopia is a rare complaint, ${ }^{7}$ but this is not borne out by these figures. It was commoner than expected accounting for $25.1 \%$ of all patients presenting with diplopia and $0.35 \%$ of all new casualties. Monocular diplopia arises when two images of the same object are seen by one eye. Typically the secondary image is fainter than the primary image and may be continuous with it or separated from it. ${ }^{9}$ In some cases triplopia or polyopia may be experienced by the patient. The second image may be vertically or horizontally separated from the primary image, and when due to lenticular causes the diplopia is typically vertical. ${ }^{7,10}$

There is no concensus in the literature as to the commonest cause of monocular diplopia. Despite the claim that psychogenic factors are the most common cause of monocular diplopia $^{8}$ in this study a genuine cause was found in $88.4 \%$ of cases. In the eight cases for which no cause could be identified the symptoms spontaneously resolved within six weeks in four patients and the other four were lost to follow up. Although several authors have stated that lenticular induced monocular diplopia is the most common form, ${ }^{9,11-13}$ Amos cautioned 
Table II. Causes of Binocular Diplopia

\section{SPECTACLES}

Bifocal intolerance

CONVERGENCE/ACCOMMODATION PROBLEMS

Convergence insufficiency

Accommodative spasm

BREAKDOWN OF EXISTING OCULAR MUSCLE IMBALANCE

Decompensating exophoria

Decompensating esophoria

G OCULAR MUSCLE IMBALANCE

TRAUMA

Blow out fracture

Blunt trauma

Penetrating injury

Post surgical

Post concussion syndrome

MUSCULAR

'Squint'

Thyroid

Myasthenia gravis

Orbital myositis

ORBITAL

Frontoethmoidal sinusitis

Orbital cellulitis

Orbital tumour

INFRANUCLEAR LESIONS

III Nerve palsy

Diabetes mellitus

Vascular

Pituitary tumour

Orbital pseudotumour

Post traumatic

Inferior rectus weakness associated with herpes zoster ophthalmicus

Unknown

IV Nerve palsy

Congential

Diabetes mellitus

Vascular

Herpes zoster

Trauma

Unknown

No follow up

VI Nerve palsy

Diabetes mellitus

Vascular

Multiple sclerosis

Herpes zoster

Cerebral tumour

Benign intracranial hypertension

Unknown

No follow up

SUPRANUCLEAR LESIONS

Internuclear ophthalmoplegia

Brain stem ischaemia

Skew deviation

'Migraine'

Wernicke's encephalopathy

HEMIFIELD SLIP

NO CAUSE ESTABLISHED 
most frequent cause of this symptom. However, in this study I identified only one patient with symptoms induced by low myopia and none with physiological monocular diplopia. Spectacle induced diplopia was most frequently seen with bifocals. Monocular diplopia in the presence of low refractive errors is thought to be the result of minor optical irregularities, principally spherical aberation. ${ }^{7,15}$ Coffeen and Guyton found that this type of diplopia could be readily induced in. nine of 11 normal eyes examined. ${ }^{15}$

Lenticular abnormalities were the commonest cause of monocular diplopia in this series and accounted for $27(39.1 \%)$ cases. These are most commonly fluid clefts within the lens, early nuclear sclerosis, cortical spokes or posterior subcapsular lens opacities. Physiological diplopia is also induced by lenticular changes, probably due to refractive index changes within the lens. ${ }^{10,11}$ In the four cases related to decentred intraocular lenses the symptoms responded to pupillary constriction with weak pilocarpine solution. It is interesting that although dislocated or subluxed lenses are a theoretical cause of monocular diplopia there have been no reports of this in the literature. Corneal disease was the second commonest cause of monocular diplopia and resulted from a wide variety of pathological processes, but the common factor was either epithelial irregularity, irregular corneal astigmatism or corneal scarring.

Although some authors have described retinal conditions as a possible cause of monocular diplopia only three true cases have been reported in the literature.$^{8,16,17}$ In this series four patients had macular pathology accounting for their symptoms, but the double vision they described was invariably associated with metamorphopsia.

The frequency of monocular diplopia in this study emphasises the importance of thorough clinical assessment in patients presenting with diplopia. They should first be questioned as to whether or not the symptoms resolve when one eye is occluded. Having made the diagnosis of monocular diplopia one must then determine if the cause is optical. If it is related to extraocular factors it will resolve when the spectacles or contact lenses are removed. Optical factors of ocular origin will usually be eliminated with a pin-hole test. ${ }^{7,12}$
Binocular diplopia occurred more frequently than monocular diplopia and accounted for $74.9 \%$ of all of patients in this study. The commonest cause was infranuclear cranial nerve palsies (81 patients: $29.5 \%$ of all causes and $39.3 \%$ of binocular causes). This figure is similar to other studies of patients presenting with binocular diplopia. ${ }^{3,4}$ However, Trimble in a similar patient population reported a much higher incidence of cranial nerve palsies $(73 \%),{ }^{6}$ as did Nolan in a study from a general ophthalmic outpatient clinic $(62.8 \%)^{1}$

The high incidence of traumatic cases has not been observed in other studies. Patients presenting to general emergency departments with orbital trauma usually do so as a result of the injury rather than diplopia. In contrast in this study many patients presented some days after their initial trauma when swelling had resolved and diplopia had become a prominent symptom.

Five patients developed binocular diplopia following cataract surgery. In three of these there was a manifest strabismus prior to surgery and the patients developed symptoms following cataract extraction. Two patients had fusional loss after removal of longstanding traumatic cataracts, the mechanism of this has recently been discussed in the literature. ${ }^{18}$ Thyroid eye disease and myasthenia gravis are important causes of diplopia and may coexist in some patients. Both conditions are associated with other ocular and systemic features and can usually be diagnosed clinically. In some cases a tensilon test, biochemical or radiological investigations may be needed to confirm the diagnosis.

Previous studies of patients with diplopia from orthoptic departments have shown a high incidence of convergence insufficiency (11\% and $33 \%$ ), and decompensating heterophorias $(11 \%$ and $17 \%)$. These figures are higher than in the present study and reflect the type of patient seen in orthoptic departments compared with that seen in casualty departments.

Important but less common causes of diplopia included orbital disease and supranuclear lesions. Because of the potentially serious nature of these conditions such patients require immediate investigation and treatment. 
In summary, the study has shown that diplopia is a common symptom which may be the presenting feature of a wide range of ocular and neurological disorders.

Monocular diplopia was found to be much more common than previously reported and a cause was identifiable in the majority of cases. Binocular diplopia occurred more frequently than monocular diplopia and was most often the result of an infranuclear cranial nerve palsy. Patients with double vision usually present to an ophthalmologist and in most cases the diagnosis can be established by careful clinical assessment of patients. Appropriate investigations can confirm the diagnosis and establish the underlying aetiology avoiding unnecessary, time consuming and expensive investigations.

I should like to thank the nursing staff and registrars in the Casualty Department for their help during the study, and the consultants who allowed me to report their patients. I should also like to thank Mr. A. Moore, Mr. J. P. Lee and particularly Mr. J. Elston who gave me valuable advice throughout the study. Key words: Double vision, Monocular diplopia, Binocular diplopia.

\section{References}

${ }^{1}$ Nolan J: Diplopia. Br J Ophthalmol 1968, 52: 166-71.

${ }^{2}$ Boyd T, Ridgway C, Budd G: Childhood strabismus as a cause of persistent diplopia in adolescents and adults. Can J Ophthalmol 1966, 1: 199-205.
${ }^{3}$ Brown S, Atkins I, Doyle M, et al. Symptom producing diplopia. Am Orthopt J 1983, 33: 105-10.

+ Yapp JMS: Diplopia as a presenting symptom. $\mathrm{Br}$ Orthopt J 1973, 30: 52-65.

${ }^{5}$ Trimble RB: Diplopia as a presenting sign of neoplasia. Trans Ophthalmol Soc UK 1980, 100: 498-500.

${ }^{6}$ Trimble RB and Kelly V: Diplopia as a presenting symptom: A prospective study. Proc IV Int Orthop Congr, Berne 1979, 91-4.

${ }^{7}$ Amos JF: Diagnosis and management of monocular diplopia. J Am Optom Assoc 1986, 53: 101-15.

${ }^{8}$ Lepore FE and Yarian DL. Monocular diplopia of retinal origin. J Clin Neuro-ophthalmol 1986, 6: 181-3.

${ }^{9}$ Records RE: Monocular diplopia. Surv Ophthalmol 1980, 24: 303-6.

${ }^{10}$ Rubin ML. The woman who saw too much. Surv Ophthalmol 1972: 16: 382-3.

${ }^{11}$ Fincham EF: Monocular diplopia. Br J Ophthalmol 1963, 47: 705-12.

${ }^{12}$ Lawton-Smith J: Monocular diplopia. (Editorial). $J$ Clin Neuro-ophthalmol 1986, 6: 184-5.

${ }^{1.3}$ Lee J and Elston J: Diplopia. Br J Hosp Med 1985, 13-20.

${ }^{14}$ Scott AB: Diplopia in myopia. Surv Ophthalmol 1974, 19: 166-8.

${ }^{15}$ Coffeen P and Guyton DL: Monocular diplopia accompanying ordinary refractive errors. Am J Ophthalmol 1988, 105: 451-9.

${ }^{16}$ Evans J. Seeing double. Med Times Long Island Med J 1933, 62: 132-4.

${ }^{17}$ Negre M: Un case de vaste desinsertion avec inferoversion de la retine. Diplopie monoculaire secondaire. Bull Soc d'Ophthal de Paris 1931, 42: 196-201.

${ }^{18}$ Pratt-Johnson JA, Tillson G: Intractable diplopia after vision restoration in unilateral cataract. $A m$ J Ophthalmol 1989, 107: 23-6. 